\title{
The potential biomarkers in predicting pathologic response of breast cancer to three different chemotherapy regimens: a case control study
}

\author{
Linbo Wang ${ }^{* 1}$, Zhinong Jiang1, Meihua Sui ${ }^{2}$, Jianguo Shen ${ }^{1}$, Chaoyang Xu ${ }^{1}$ \\ and Weimin Fan*2
}

\author{
Address: ${ }^{1}$ Department of Surgical Oncology, Sir Run Run Shaw Hospital, Zhejiang University College of Medicine, Hangzhou, PR China and \\ 2Department of Pathology and Laboratory Medicine, Medical University of South Carolina, Charleston, South Carolina, USA \\ Email: Linbo Wang* - drlinbowang@hotmail.com; Zhinong Jiang - jzn1008@163.com; Meihua Sui - suim@musc.edu; \\ Jianguo Shen - shenjianguo76623@hotmail.com; ChaoyangXu - xuchaoyang2001@126.com; Weimin Fan* - fanw@musc.edu \\ * Corresponding authors
}

Published: II July 2009

BMC Cancer 2009, 9:226 doi:10.1 186/147|-2407-9-226

This article is available from: http://www.biomedcentral.com/I47I-2407/9/226

(c) 2009 Wang et al; licensee BioMed Central Ltd.

This is an Open Access article distributed under the terms of the Creative Commons Attribution License (http://creativecommons.org/licenses/by/2.0), which permits unrestricted use, distribution, and reproduction in any medium, provided the original work is properly cited.

\begin{abstract}
Background: Preoperative chemotherapy (PCT) has become the standard of care in locally advanced breast cancer. The identification of patient-specific tumor characteristics that can improve the ability to predict response to therapy would help optimize treatment, improve treatment outcomes, and avoid unnecessary exposure to potential toxicities. This study is to determine whether selected biomarkers could predict pathologic response (PR) of breast tumors to three different PCT regimens, and to identify a subset of patients who would benefit from a given type of treatment.
\end{abstract}

Methods: I I 8 patients with primary breast tumor were identified and three PCT regimens including DEC (docetaxel+epirubicin+cyclophosphamide), VFC (vinorelbine/vincristine+5fluorouracil+cyclophosphamide) and EFC (epirubicin+5-fluorouracil+cyclophosphamide) were investigated. Expression of steroid receptors, HER2, P-gP, MRP, GST-pi and Topo-II was evaluated by immunohistochemical scoring on tumor tissues obtained before and after PCT. The PR of breast carcinoma was graded according to Sataloff's classification. Chi square test, logistic regression and Cochran-Mantel-Haenszel assay were performed to determine the association between biomarkers and $P R$, as well as the effectiveness of each regimen on induction of PR.

Results: There was a clear-cut correlation between the expression of ER and decreased PR to PCT in all three different regimens $(p<0.05)$. HER2 expression is significantly associated with increased PR in DEC regimen $(p<0.05)$, but not predictive for PR in EFC and VFC groups. No significant correlation was found between biomarkers PgR, Topo-II, P-gp, MRP or GST-pi and PR to any tested PCT regimen. After adjusted by a stratification variable of ER or HER2, DEC regimen was more effective in inducing PR in comparison with VFC and EFC regimens.

Conclusion: ER is an independent predictive factor for PR to PCT regimens including DEC, VFC and EFC in primary breast tumors, while HER2 is only predictive for DEC regimen. Expression of PgR, Topo-II, PgP, MRP and GST-pi are not predictive for PR to any PCT regimens investigated. Results obtained in this clinical study may be helpful for the selection of appropriate treatments for breast cancer patients. 


\section{Background}

Chemotherapy is used in both inoperable and operable breast tumors, to promote tumor shrinkage and to render these tumors treatable by radical mastectomy or radiotherapy, or with the objective of down staging the tumor so that breast-conserving surgery could become a viable alternative to radical mastectomy. In recent years, preoperative chemotherapy has become the standard of care in locally advanced breast cancers $[1,2]$. Previous clinical trials have shown that preoperative chemotherapy allows regression of the tumor in order to avoid mastectomy and to treat clinically undetectable micrometastatic disease. In addition, preoperative chemotherapy permits the assessment of response of the primary tumor to a particular chemotherapy regimen and provides an early opportunity to alter the agents if the tumor appears clinically resistant.

Although several different classes of chemotherapeutic drugs are applied in the preoperative setting include taxanes, anthracyclines and vinca alkaloids, etc., there are currently no standard regimens or protocols for preoperative chemotherapy in breast cancer patients [2]. On the other hand, a significant proportion of breast tumors are resistant to chemotherapeutic agents. As a result, drug resistance has become the major cause of cancer chemotherapy failure and is largely responsible for breast cancer mortality. Therefore, the identification of patient-specific tumor characteristics that can improve the ability to predict response to therapy would help optimize treatment, improve treatment outcomes, and avoid unnecessary exposure to potential toxicities.

However, although the factors predicting response of breast tumors to hormonal therapy are known [3-6], relatively few studies to elucidate factors influencing the response of patients to treatment of combination chemotherapy have been undertaken. Previous studies on the response to chemotherapy in breast cancer either focused on single chemotherapy regimen or single class of anticancer drugs [7-9], or the combined treatment of chemotherapy and hormonal therapy $[10,11]$, which may provide limited information or blunt the effectiveness of chemotherapy alone. Thus, more clinical studies investigating the potential predictive or prognostic biomarkers for breast cancer chemotherapy are needed.

Patients who exhibited pathologic complete response (pCR) to chemotherapy showed better progression-free survival and overall survival compared to those with residual tumor $[12,13]$. Therefore, $\mathrm{pCR}$ is now recognized as an independent prognostic factor of patients with breast carcinoma. To assess whether the selected biomarkers may play a role in predicting pathologic response of primary breast tumors to preoperative chemotherapy, and to identify a subset of patients who would benefit from a given type of preoperative treatment, 118 patients with operable breast cancer treated with three different preoperative chemotherapy regimens have been evaluated in the present study. These patients with primary breast tumors were subjected to three different chemotherapy regimens before surgery, including DEC (docetaxel+epirubicin+cyclophosphamide), VFC (vinorelbine/vincristine+5-fluorouracil+cyclophosphamide) and EFC (epirubicin+5-fluorouracil+cyclophosphamide). The molecular makers measured in this study include estrogen receptor (ER), progesterone receptor (PgR), human epidermal growth factor receptor 2 (HER2), P-glycoprotein (P-gp), multidrug resistance-related protein (MRP), glutathione S-transferase pi (GST-pi) and topoisomerase-II (Topo-II), based on their potential roles in modulating tumor biology and sensitivity or resistance to anticancer treatments [14-16].

\section{Methods \\ Patient selection}

One hundred and eighteen consecutive breast cancer patients presented at the Zhejiang University Sir Run Run Shaw Hospital, Hangzhou, China, who received preoperative chemotherapy during the period of February 2002 to January 2007 were enrolled in this study. Patients with radiotherapy or endocrine therapy before surgery, and those whose paraffin-embedded tissue from the biopsy was insufficient to allow a pathologic diagnosis and evaluation of biomarkers, were excluded. The median age was 49 years (range, $30-84$ years). Thirty-eight patients (32\%) were postmenopausal. The distribution of cases according to tumor size, clinical node status, and menopausal status

Table I: Patient characteristics $(n=1$ I 8)

\begin{tabular}{lc}
\hline Characteristic & Value \\
\hline Age & \\
Median & 49 \\
Range & $30-84$ \\
Menopausal Status & \\
$\quad$ Premenopausal & $80(68 \%)$ \\
Postmenopausal & $38(32 \%)$ \\
Histologic Type & \\
Ductal Carcinoma & $100(84.7 \%)$ \\
Lobular Carcinoma & $6(5.1 \%)$ \\
Unclassified & $12(10.2 \%)$ \\
Clinical Primary Tumor & \\
TI & $19(16.1 \%)$ \\
T2 & $19(16.1 \%)$ \\
T3 & $74(62.7 \%)$ \\
T4 & $6(5.1 \%)$ \\
Chemotherapeutic Regimens* & \\
DEC & $58(49.2 \%)$ \\
EFC & $37(31.4 \%)$ \\
VFC & $23(19.5 \%)$ \\
\hline * D docetaxel, E epirubicin, C cyclophosphamide, F 5-Fluorouracil, V \\
vinorelbine or vincristine
\end{tabular}


is shown in Table 1 . This study was approved by the ethical committee of Sir Run Run Shaw Hospital. Informed consents from patients were obtained for the use of paraffin-embedded tumor tissue.

\section{Treatment plan}

Preoperative chemotherapy was started within 1 or 2 days of diagnosis. Fifty-eight patients were treated with DEC regimen containing $75 \mathrm{mg} / \mathrm{m}^{2}$ docetaxel i.v. on day 1,60 $\mathrm{mg} / \mathrm{m}^{2}$ epirubicin i.v. on day 1 and $500 \mathrm{mg} / \mathrm{m}^{2}$ cyclophosphamide i.v. on day 1 every three weeks for 2 cycles. Thirty-seven patients received EFC regimen containing epirubicin 60 (or 70 ) mg/m² i.v. on days 1 and 8,5 -fluorouracil $500 \mathrm{mg} / \mathrm{m}^{2} i . v$. on day 1 and cyclophosphamide $500 \mathrm{mg} / \mathrm{m}^{2}$ i.v. on day 1 every three weeks for 2 cycles. Twenty-three patients were treated with VFC regimen containing vinorelbine $25 \mathrm{mg} / \mathrm{m}^{2} i . v$. or vincristine $1.3 \mathrm{mg} / \mathrm{m}^{2}$ i.v. on day 1 , cyclophosphamide $375 \mathrm{mg} / \mathrm{m}^{2}$ i.v. on days 2,4 and 6 , and 5 -fluorouracil $375 \mathrm{mg} / \mathrm{m}^{2}$ i.v. on days 1,3 and 5 every three weeks for 2 cycles. Table 2 shows the detailed employment of the above preoperative chemotherapy regimens in patients with primary breast tumors. Breast cancer surgery was performed after 2 weeks of the last cycle of chemotherapy.

\section{Assessment of pathologic response}

Tumor tissues were obtained 2-3 days prior to chemotherapy via core biopsy or during the surgery. Tumor samples obtained before and after chemotherapy were fixed with $10 \%$ buffered-formalin, embedded in paraffin and stained with hematoxylin and eosin. The pathologic response of breast carcinomas was graded according to Sataloff's classification (primary site response classification) [17]. Given the nature of their results and using the same guidelines, we re-named the four categories in Sataloff's classification into two groups: Group I as responders (major pathologic response) and Group II as nonresponders (minor or no response), both of which contain two subgroups. Therefore, patients were divided into the following four groups: Group Ia, total or near total therapeutic effect; Group Ib, subjectively more than 50\% therapeutic effect but less than total or near total; Group IIa, less than 50\% therapeutic effect, but effect evident; and Group IIb, no therapeutic effect. Pathologic response rate (pRR) was defined as a ratio of responders to total number of tumors (responders plus non-responders).

\section{Immunohistochemical assay}

Immunohistochemical staining was completed in the pathology laboratory of Sir Run Run Shaw Hospital. The

Table 2: Preoperative chemotherapy regimens employed in patients

\begin{tabular}{|c|c|c|c|c|}
\hline Biomarker & DEC $(n=58)$ & $\operatorname{EFC}(n=37)$ & VFC $(n=23)$ & All Patients $(n=118)$ \\
\hline \multicolumn{5}{|l|}{ ER } \\
\hline negative & 28 & 10 & 14 & 52 \\
\hline positive & 30 & 27 & 9 & 66 \\
\hline positive rate(\%) & 51.7 & 73.0 & 39.1 & 55.9 \\
\hline \multicolumn{5}{|l|}{$P R$} \\
\hline negative & 31 & 19 & 12 & 62 \\
\hline positive & 27 & 18 & 11 & 56 \\
\hline positive rate(\%) & 46.6 & 48.6 & 47.8 & 47.5 \\
\hline \multicolumn{5}{|l|}{ HER2 } \\
\hline negative & 24 & 16 & 9 & 49 \\
\hline positive & 34 & 21 & 14 & 69 \\
\hline positive rate(\%) & 58.6 & 56.8 & 60.9 & 58.5 \\
\hline \multicolumn{5}{|l|}{ P-gP } \\
\hline negative & 44 & 30 & 19 & 93 \\
\hline positive & 14 & 7 & 4 & 25 \\
\hline positive rate(\%) & 24.1 & 18.9 & 17.4 & 21.2 \\
\hline \multicolumn{5}{|l|}{ MRP } \\
\hline negative & 26 & 13 & 9 & 48 \\
\hline positive & 32 & 24 & 14 & 70 \\
\hline positive rate(\%) & 55.2 & 64.9 & 60.9 & 59.3 \\
\hline \multicolumn{5}{|l|}{ GST-pi } \\
\hline negative & 35 & 23 & 13 & 71 \\
\hline positive & 23 & 14 & 10 & 47 \\
\hline positive rate(\%) & 39.7 & 37.8 & 43.5 & 39.8 \\
\hline \multicolumn{5}{|l|}{ Topo-II } \\
\hline negative & 23 & 14 & 7 & 44 \\
\hline positive & 35 & 23 & 16 & 74 \\
\hline positive rate(\%) & 60.3 & 62.2 & 69.6 & 62.7 \\
\hline
\end{tabular}

* D docetaxel, E epirubicin, C cyclophosphamide, F 5-Fluorouracil, V vinorelbine or vincristine 
sections cut at 4-6 $\mu \mathrm{m}$ were deparaffinized in xylene and rehydrated in a series of graded ethanol. The slides were immersed in $10 \mathrm{mM}$ citrate buffer ( $\mathrm{pH}$ 6.0) and boiled for 15 minutes in a pressure cooker with the lid on for antigen retrieval. The slides were let cool down in the box at room temperature for $20 \mathrm{~min}$ and then washed with phosphate buffered saline (PBS). Endogenous peroxidase was blocked with $0.3 \% \mathrm{H}_{2} \mathrm{O}_{2}$ in methanol for $10 \mathrm{~min}$, and nonspecific binding was blocked by preincubation with $2 \%$ fetal calf serum in PBS with $0.1 \%$ sodium azide for 30 minutes [18].

The panel of markers detected by IHC was as followings: ER (Neomarker, rabbit-mAb, clone sp1), PgR (Lab Vision, rabbit-mAb, clone sp2), HER2 (Lab Vision, rabbit-mAb, clone sp3), P-gp (ZSGB-Bio, mouse-mAb, clone c219), MRP (GeneTech, mouse-mAb, clone 33A6), GST-pi (Dako, rabbit-polyAb), Topo-II (Dako, mouse-mAb, clone KI-S1). After washing three times in PBS, the slides were incubated with the EnVision-HRP complex (Dako, Carpinteria, CA) for $60 \mathrm{~min}$. Finally, the slides were visualized with diaminobenzidine (Dako) and counterstained with hematoxylin. For substitute negative controls, the primary antibody was replaced with PBS. Positive controls included breast or colonic carcinomas known to exhibit high levels of each marker.

\section{Scoring of immunohistochemical assay}

Immunohistochemical analysis reported in this study was carried out in a single laboratory. All slides were reviewed and scored independently by two pathologists without knowledge of the demographic or treatment response information. ER, PgR and Topo-II were scored as positive when at least $10 \%$ of the carcinoma cell nuclei were immunoreactive [7]. The intensity of membrane staining of HER2 was evaluated according to the criteria set forth by the DAKO Hercep Test: score $0=$ no or up to $10 \%$ membrane staining; score $1+=$ partial and/or faint membrane staining in more than $10 \%$ of tumor cells; score $2+$ = weak to moderate complete membrane staining in more than $10 \%$ of tumor cells; and score $3+=$ strong complete membrane staining in more than $10 \%$ of tumor cells [7]. Ascore of 0 and $1+$ for HER2 was considered negative, whereas $2+$ and $3+$ were considered positive. We defined ER negative, PR negative and HER2 negative as triple-negative phenotype, regardless of the expression of other biomarkers [19]. Other combinations of ER, PgR and HER2 expression was considered non-triple negative phenotype. The evaluation criteria for P-gp, MRP and GST-pi expression were interpreted based on the percentage of positive tumor cells: score $0=$ no staining; score $1+=$ up to $25 \%$ staining; score $2+=25-50 \%$ staining; and score $3+$ $=$ more than $50 \%$ staining of tumor cells [20]. Ascore of 0 was considered negative, and all other scores were considered positive.

\section{Statistical analysis}

Descriptive studies were performed with SPSS 15.0 for windows $[10,11]$. Chi square test (or Fisher's exact test) and logistic regression were performed in univariate and multivariate analysis, respectively, to determine the relationship between expression of biomarkers and pathologic response. Chi square test was also used for association between different biomarkers. The relationship between different chemotherapeutic regimen and pathologic response was assessed with Cochran-MantelHaenszel chi-square test adjusted for the effect of a stratification variable ER. A $p$ value of $<0.05$ was considered significant. Two-sided statistical tests were used in all the above analyses.

\section{Results \\ Response to preoperative chemotherapy}

The pathologic responses were evaluated in all 118 patients at the end of two cycles of preoperative chemotherapy administration. According to the criteria defined above, 26 of $118(22.0 \%)$ patients had Ia (total/near total therapeutic effect); 14 of 118 (11.9\%) patients had Ib (subjectively $>50 \%$ therapeutic effect but $<$ total/near total therapeutic effect); 23 of 118 (19.5\%) patients had IIa ( $<50 \%$ therapeutic effect); and 55 of 118 (46.6\%) patients had IIb (no therapeutic effect). For further analysis, patients treated with each combination chemotherapeutic regimen were divided into two categories based on the response to treatment: responders $(\mathrm{Ia}+\mathrm{Ib})$ and nonresponders (IIa $+\mathrm{IIb})$. There were 25 responders of 58 $(43.1 \%)$ in DEC regimen group, 6 responders of 37 (16.2\%) in EFC regimen, and 9 responders of 23 (39.1\%) in $\mathrm{VFC}$ regimen group.

\section{Evaluation of immunostaining and the correlation among biomarkers}

Expression of biomarkers was immunohistochemically determined in biopsy specimens obtained before preoperative chemotherapy. Positive ER, PgR and HER2 immunostainings were noted in 55.9\%, $47.5 \%$ and $58.5 \%$ of the specimens, respectively. P-gp, MRP, GST-pi and TopoII positive staining were observed in $21.1 \%$, $60.2 \%$, $39.8 \%$ and $62.7 \%$ of tumors, respectively. Fifteen of all 118 specimens presented triple negative phenotype (negative ER, PgR and HER2). As shown in Table 3, statistically significant correlations on expression of certain biomarkers were observed by using Chi square test. In brief, a strong direct correlation was found between ER and PgR receptor status. ER negative tumors were usually PgR negative, and only 6 tumors showed PgR positive in $52 \mathrm{ER}$ negative tumors. In contrast, ER expression was inversely correlated with HER2, GST-pi and Topo-II. For example, $71.2 \%$ of ER negative tumors showed HER2 positive. In addition, significantly direct correlation was also observed between HER2 and Topo-II, as well as between GST-pi and MRP (see Table 3). 
Table 3: Statistically significant correlations between biomarkers

\begin{tabular}{ccc}
\hline Biomarkers Evaluated & R Value & p Value ${ }^{* *}$ \\
\hline ER to PR & 0.638 & 0.000 \\
ER to HER2 & -0.228 & 0.013 \\
ER to GST-pi & -0.254 & 0.005 \\
ER to Topo-II & -0.261 & 0.004 \\
GST-pi to MRP & 0.251 & 0.006 \\
HER2 to Topo-II & 0.204 & 0.027 \\
\hline
\end{tabular}

$*^{*} p<0.05$ is considered statistically significant

\section{Correlation between expression of biological markers and pathologic response}

Table 4 summarizes the results of univariate analyses for the relationship between pathologic response and biomarkers. Moreover, the multivariate analysis was made by using the stepwise forward logistic regression model for the co-variables including ER, PgR, HER2, P-gp, MRP, GST-pi, Topo-II and three different chemotherapeutic regimens. The statistically significant independent factor in multivariate analysis was listed in Table 5.

In univariate analysis and logistic regression analysis (Tables 4 and 5), there was a clear-cut correlation between the expression of ER and decreased pathologic response to preoperative chemotherapy in all three different regimens $(p<0.05)$. PgR was correlated with pathologic response to DEC regimen but not other two regimens in univariate analysis. However, no significant correlation between PgR and any chemotherapy regimen was found in multivariate analysis $(p>0.05)$. HER2 expression was significantly associated with increased pathologic response rate in breast tumors treated with DEC regimen in both univariate and multivariate analyses, suggesting that HER2 was an independent predictive factor for DEC treatment $(p=$ $0.026)$. However, no significant predictive effect of HER2 was found in EFC and VFC regimens, although HER2 negative tumors showed slightly higher pathologic response rate than positive tumors $(p>0.05$, see Table 4$)$. Tumors with triple negative phenotype (negative ER, PgR and HER2) achieved significantly higher pathologic response rate than non-triple negative phenotype $(60.0 \%$ versus $30.1 \%, p=0.022)$. Interestingly, in ER negative tumors, the pathologic response rate of triple negative tumors was similar with non-triple negative tumors $(60.0 \%$ versus $62.2 \%, p=0.885$ ).

A correlation between Topo-II expression and greater pathologic response to preoperative chemotherapy in DEC regimen group was observed in univariate analysis ( $p$ $=0.034)$ but not in multivariate analysis $(p=0.202)$. No statistical significance between Topo-II expression and pathologic response was achieved in EFC and VFC regi-

Table 4: Univariate analysis for pRR and biomarkers\#

\begin{tabular}{|c|c|c|c|c|c|c|}
\hline \multirow[t]{2}{*}{ Biomarker } & \multicolumn{2}{|c|}{$D_{E C} n=58$} & \multicolumn{2}{|c|}{$E F C n=37$} & \multicolumn{2}{|c|}{ VFC $n=23$} \\
\hline & pRR (\%) & $\mathbf{p}^{* *}$ & pRR (\%) & $\mathbf{p}^{* *}$ & pRR (\%) & $\mathbf{p}^{* *}$ \\
\hline \multicolumn{7}{|l|}{ ER } \\
\hline negative & 67.9 & $0.000 * *$ & 50.0 & $0.003 * *$ & 57.1 & $0.040^{* * *}$ \\
\hline positive & 20.0 & & 3.7 & & 11.1 & \\
\hline \multicolumn{7}{|l|}{$P R$} \\
\hline negative & 58.1 & $0.014 * *$ & 26.3 & 0.180 & 58.3 & 0.089 \\
\hline positive & 25.9 & & 5.6 & & 18.2 & \\
\hline \multicolumn{7}{|l|}{ HER2 } \\
\hline negative & 16.7 & $0.001 * *$ & 25.0 & 0.371 & 55.6 & 0.383 \\
\hline positive & 61.8 & & 16.2 & & 28.6 & \\
\hline \multicolumn{7}{|l|}{ P-gP } \\
\hline negative & 38.6 & 0.223 & 13.3 & 0.315 & 42.1 & 1.000 \\
\hline positive & 57.1 & & 28.6 & & 25.0 & \\
\hline \multicolumn{7}{|l|}{ MRP } \\
\hline negative & 32.0 & 0.137 & 7.7 & 0.394 & 22.2 & 0.228 \\
\hline positive & 51.5 & & 20.8 & & 50.0 & \\
\hline \multicolumn{7}{|l|}{ GST-pi } \\
\hline negative & 42.9 & 0.963 & 8.7 & 0.173 & 30.8 & 0.417 \\
\hline positive & 43.5 & & 28.6 & & 50.0 & \\
\hline \multicolumn{7}{|l|}{ Topo-II } \\
\hline negative & 26.1 & $0.034 * *$ & 6.7 & 0.368 & 42.9 & 1.000 \\
\hline positive & 54.3 & & 22.7 & & 37.5 & \\
\hline
\end{tabular}


Table 5: Multivariate analysis for pRR and biomarkers\#

\begin{tabular}{cccc}
\hline Treatment* & Independent Factor & OR & $\boldsymbol{p ~ V a l u e * *}$ \\
\hline DEC $(n=58)$ & ER & 5.2 & $<0.001$ \\
DEC $(n=58)$ & HER2 & 0.2 & 0.026 \\
EFC $(n=37)$ & ER & 26.0 & 0.007 \\
VFC $(n=23)$ & ER & 10.7 & 0.047 \\
All patients $(n=118)$ & ER & 11.6 & $<0.001$ \\
\hline
\end{tabular}

\# pRR: pathologic response rate

* D docetaxel, E epirubicin, C cyclophosphamide, F 5-Fluorouracil, V vinorelbine or vincristine

$* * p<0.05$ is considered statistically significant

mens ( $p=0.368$ and 1.000, respectively) in univariate analysis. Moreover, no significant correlation was found between biomarkers P-gp, MRP or GST-pi and pathologic response to preoperative treatment of any chemotherapy regimen investigated in this study.

\section{Histological features of the primary breast tumors with different estrogen receptor status to preoperative chemotherapy}

The histological effects of preoperative chemotherapy in primary breast tumors with different ER status were evaluated by comparing biopsy and surgical samples taken before and after chemotherapy. Tumor samples were fixed in formalin for hematoxylin and eosin (H\&E) staining and immunohistochemical staining with anti-ER $\alpha$ antibody using standard protocols. Various pathological changes were observed after preoperative chemotherapy, which mainly included coagulative necrosis of tumor tissue, fibrosis/hyalinization, as well as mixed inflammatory infiltrate. Chemotherapy could also induce cytological changes in tumor cells in cytoplasm and/or nuclear. For example, the cytoplasm of tumor cells was either intensely eosinophilic or clear with a vacuolated or foamy appearance. Enlarged or bizarre nuclear with clumped chromatin may be recognized. Furthermore, the volume of residual tumor varied based on different chemotherapeutic effects. In our study, most ER-negative breast tumors showed significant better pathologic response to chemotherapy than ER-positive tumors. As shown in Fig. 1, there were still many survival tumor cells with ER positivity after the preoperative chemotherapy of EFC regimen in ER-positive primary breast tumors. However, the ER-negative primary tumor exhibited significantly improved pathologic response to EFC treatment, which was demonstrated by the dramatically reduced volume of residual tumor, as well as the remarkable fibrosis and hyalinization of tissue after the treatment.

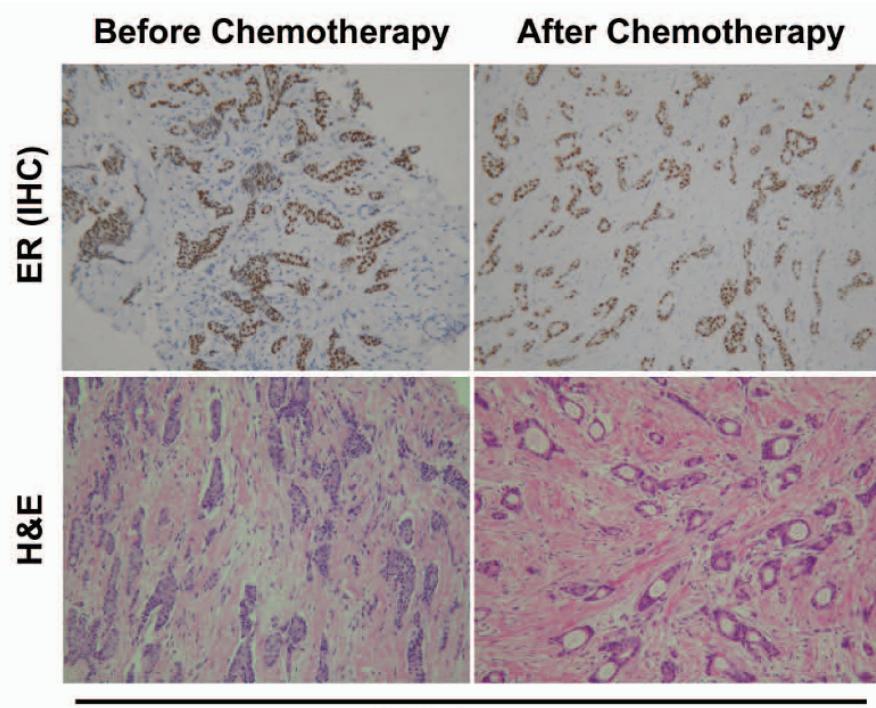

ER+ tumor

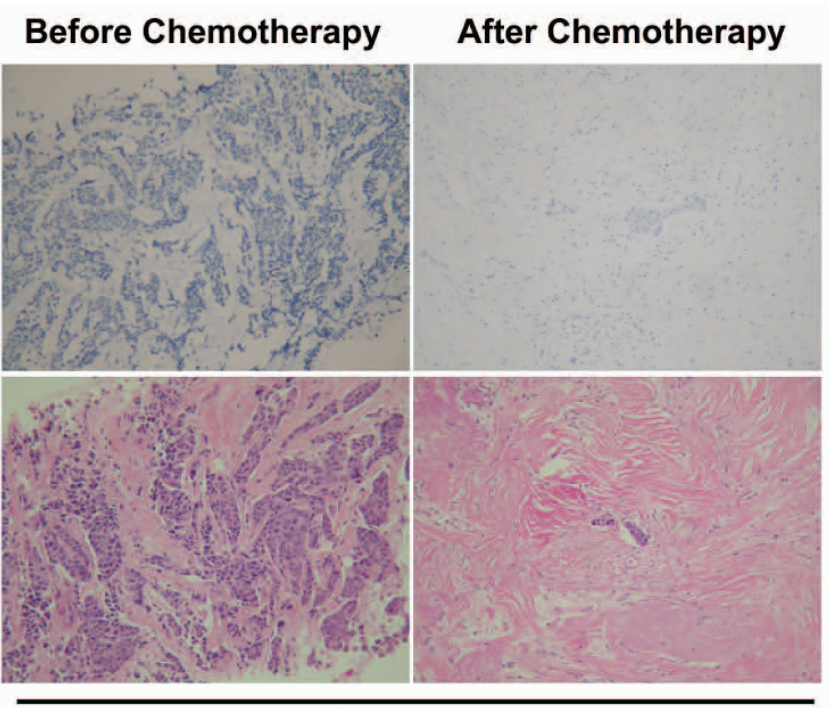

ER- tumor

\section{Figure I}

Immunohistochemical staining with ER (upper panel) and H\&E staining (lower panel). Stainings were performed on formalin-fixed, paraffin-embedded tissue sections of invasive ductal breast carcinoma, and photographed before and after preoperative chemotherapy treatment of EFC regimen, by using a Zeiss Axioskop40 Microscope and a Nikon E4500 camera. In ER+ tumor sample (left), tumor cells arranged in glands and clusters still showed active growth after the EFC chemotherapy treatment. Both core needle biopsy specimen and surgical specimen showed diffuse nuclear staining for estrogen receptor (diaminobenzidine chromogen). However, in ER- tumor sample (right), only a small nest of tumor cells remained survival after EFC treatment, with prominent stromal fibrosis and hyalinization. ER staining was negative in both biopsy and surgical specimens. Data are representative of at least three separate experiments. Magnification: $\times 100$. 


\section{Correlation between chemotherapy regimen and pathologic responses}

As described above, our results indicate that ER is an independent predictive factor for all three different chemotherapy regimens investigated in this study. Because the positive rates of ER in tumors treated with DEC and VFC regimens (51.7\% in DEC group and 39.1\% in VFC group) were obviously lower than that in EFC group $(73.0 \%)$, Cochran-Mantel-Haenszel chi-square test was utilized to compare the discrepancy of pathologic response to different chemotherapy regimens, adjusted by the effect of the stratification variable ER. After adjusted by the effect of ER, our results indicate that breast tumors treated with DEC regimen achieved higher pathologic response than tumors treated with EFC or VFC regimen (DEC versus EFC, $p=0.044$; DEC versus VFC, $p=0.030$ ), while the pathologic response rate induced by VFC regimen was similar to that induced by EFC regimen $(p=0.495)$. The difference on disparity of pathologic response rate between the above two groups appears more remarkable in ER-positive tumors than in ER-negative tumors. For example, only one of total twenty six ER-positive tumors achieved major pathologic response by treatment of EFC regimen $(\mathrm{pRR}=$ $3.7 \%$ ), and in six of thirty ER-positive tumors in DEC group achieved pathologic response $(\mathrm{pRR}=20 \%)$, although the difference did not reach statistical significance ( $p=0.105)$. While in ER-negative tumors, the difference on pathologic response between these two regimens was relatively minor (67.9\% in DEC regimen versus $50 \%$ in EFC regimen, $p=0.449)$. Moreover, after adjusted by the effect of stratification variable HER2, our data indicated that tumors treated with DEC regimen achieved significantly higher pathologic response than tumors treated with other two regimens (DEC versus EFC, $p=0.004$; DEC versus VFC, $p=0.03$ ) in HER2 positive tumors, while the pathologic response rates induced by VFC or EFC regimens were similar $(p=0.191)$. However, this superiority of DEC regimen to other two regimens was not observed in HER2 negative tumors.

\section{Discussion}

The future of cancer treatment lies in tailoring regimens to individual patients by identifying response predictors and developing novel agents. The preoperative chemotherapy is an ideal clinical setting in which to validate the relationship between tumor molecular profiling and treatment outcomes, and to optimize therapies based on observed effects on individual tumors. As a result, the evaluation of pathologic response to preoperative chemotherapy provides an ideal platform to identify the potential predictive factors of response to anticancer drugs. The present clinical study was undertaken to determine whether the expression of ER, PgR, HER2, P-gp, MRP, GST-pi and Topo-II could affect the pathologic response of primary breast tumors to three different preoperative chemother- apy regimens including DEC, VFC and EFC. A clear definition of such factors would assist in the selection of appropriate treatments for breast cancer patients.

Steroid hormone receptors, ER and PgR, are important biological markers of breast carcinoma. Their status has an established role in determining the tumor response to hormonal therapy, but their role in predicting response to chemotherapy is less clear [3-6,14]. Previous investigations evaluating the potential correlation between estrogen receptor status and pathologic response to chemotherapy have produced contradictory data: ER-positive tumors respond either better [21], less well [22-27] or the same [28-31], when compared with ER-negative tumors. Some results can be confused by the use of chemo-endocrine therapy, where the receptor-positive population may have responded to the hormonal part of the treatment $[10,11]$. The close correlation between ER and PgR observed in this study may be explained by the regulation of PgR expression by estrogen and estrogen receptor $[32,33]$. However, although PgR was correlated with pathologic response to DEC regimen in univariate analysis (Table 4), no significant correlation between PgR and any chemotherapy regimen was found in multivariate analysis (Table 4 and 5). By using both univariate analysis and logistic regression, our data indicate that ER is an independent predictive factor of the pathologic response to all three different chemotherapy regimens investigated in this study including DEC, VFC and EFC. A clear correlation between the expression of ER and decreased pathologic response to preoperative chemotherapy of all three different regimens was observed in this study (Table 4 and 5, Fig. 1). In fact, through stable transfection of estrogen receptor alpha into ER-negative human breast cancer cells, we recently demonstrated that ER may mediate breast tumor resistance to taxanes and vinca alkaloids through inhibition of drug-induced apoptotic cell death [27]. Our findings that ER-negative tumors exhibited improved pathologic response to three different combination chemotherapy regimens suggest that ER may also mediate breast cancer resistance to other chemotherapeutic drugs. Our data provide strong support for the predictive value of ER and indicate that ER seems to be more sensitive than $\mathrm{PgR}$ in predicting pathologic response to preoperative chemotherapy with DEC, VFC or EFC regimens.

Amplification and overexpression of certain oncogenes have been associated with an aggressive natural history, poor prognosis and altered sensitivity to chemotherapy (either chemosensitization or chemoresistance). Among them, one of the most promising new markers is the Her2 gene and its product (also designated c-erbB-2, c-neu) [34]. HER2 encodes a $185-\mathrm{kDa}$ transmembrane tyrosine kinase active protein, which is a component of the epidermal growth factor receptor family [34]. HER2 is over- 
expressed in 25-30\% of breast tumors. In current study, the positive rate of HER2 was $58.5 \%$, which was greater than expected. It has been shown that more advanced breast tumors usually have relatively higher expression level of HER2 [35]. Thus, we think the high proportion of HER2+ tumors may be related with the advanced clinical stage of breast cancer patients enrolled in this study. There is controversy about the overexperssion of HER2 in relation to response of breast tumor cells to chemotherapy [36-39], and the underlying mechanism involved in these processes have not been clearly elucidated. In the present study, both univariate and multivariate analysis indicated that high level of HER2 expression was significantly associated with increased pathologic response rate in breast tumors treated with DEC regimen, suggesting that HER2 might be an independent predictive factor for DEC treatment (Table 4). However, no significant predictive effect of HER2 was found in patients treated with EFC or VFC regimens ( $p>0.05$, see Table 4$)$. Triple-negative breast cancer is a recent term and refers to cancers that do not express ER, PgR and HER2 receptors. Histologically, such cancers are poorly differentiated, and most fall into the basal subgroup of breast cancers, characterized by staining for basal marker (i.e. cytokeratin) [19]. Clinically, triplenegative cancers were characterized by an aggressive clinical history, and some reports indicate that this group of breast cancer patients was associated with poor clinical outcome [19]. Very few studies have investigated the pathologic response of triple-negative breast cancers to chemotherapy [40-42]. In the present study, we found that breast tumors with triple-negative phenotype achieved significantly higher pathologic response rate than nontriple-negative phenotype (60.0\% versus $30.1 \%, p=0.022)$. Interestingly, in ER-negative tumors, the pathologic response rate of triple-negative tumors was similar with nontriple-negative tumors $(60.0 \%$ versus $62.2 \%, p=$ $0.885)$, suggesting that ER might be a more important biomarker in predicting pathologic response of breast tumors to preoperative chemotherapy than PgR and HER2.

P-gp and MRP, two ATP-dependent drug transport pumps, have been indicated to confer resistance to a number of chemotherapeutic agents such as paclitaxel, epirubicin and vinblastine [16]. The expression levels of these two transporters are $21.1 \%$ and $60.2 \%$, respectively, in our tumor samples. A significant correlation between expression of MRP and GST-pi was observed in this study (Table 3), which is consistent with previous reports indicating that GSH is necessary for MRP-mediated cellular efflux of certain drugs and detoxification of anticancer agents involves a combined action of GSTs and MRPs $[43,44]$. A significant positive correlation between P-gp expression and a poor clinical response to chemotherapy was reported in some studies $[45,46]$. However, no statis- tical association between the expression of P-gp, combined MRP and GST-pi with pathologic response to any of the three preoperative chemotherapy regimens was observed in the present study and several other reports $[43,44,47]$. On the other hand, Topo-II is an important DNA binding enzyme that modifies DNA topology. TopoII gene is located at chromosome band 17q12-21, close to the Her2 gene [15]. A significant proportion of breast cancers with Her2 amplification show simultaneous amplification of Topo-II [24], which coincides with the significant correlation between Topo-II and HER2 protein expression observed in this study (Table 3 ). Some clinical investigations have shown certain association between Topo-II and response of breast cancer to chemotherapy [48-50], but some others failed to confirm this association [51]. In our study, a statistical significance between TopoII expression and pathologic response to preoperative chemotherapy was only observed in DEC regimen in univariate analysis $(p=0.034)$, but this significance was not confirmed in multivariate analysis $(p=0.202)$.

By using a modified method of Sataloff DM to classify the pathologic response, 40 of total 118 patients in this study were classified as responders ( $\mathrm{pRR}=22.9 \%)$. Further, we evaluated the pathologic response induced by each chemotherapeutic regimen with Cochran-Mantel-Haenszel chisquare test adjusted by ER or HER2, two independent predictive biomarkers observed in this study. Our data show that after adjusted by the effect of ER, the pathologic response rate induced by DEC regimen was significantly higher than EFC and VFC regimens, while the later two regimens did not reach statistical significance. The above difference was particularly remarkable in ER-positive breast tumors than ER-negative tumors. After adjusted by the variable HER2, DEC regimen was also most effective in inducing pathologic response in HER2 positive breast tumors. These data may suggest a potential advantage in inducing relatively high pathologic response in primary breast tumors by DEC regimen compared with EFC and VFC regimens.

\section{Conclusion}

Our data show that ER is an independent predictive factor of pathologic response to three different preoperative chemotherapy regimens including DEC, EFC and VFC in breast tumors. ER-negative tumors exhibited significantly increased benefit from the above preoperative regimens compared to ER-positive tumors. HER2 is an independent predictive factor in tumors treated with DEC regimen, but not in tumors exposed to EFC or VFC regimens. Expression of PgR, Topo-II, P-gp, MRP and GST-pi were not predictive for pathologic response to any of the three preoperative chemotherapy regimens. Results obtained in this clinical study may provide valuable information on the potential correlation between selected biomarkers 
with pathologic response to preoperative chemotherapy in breast tumors, which may be helpful in the selection of appropriate treatments for breast cancer patients.

\section{Competing interests}

The authors declare that they have no competing interests.

\section{Authors' contributions}

LW and WF were responsible for the design of this study and overall analysis, and contributed equally to this work. ZJ and MS participated in the immunohistochemical staining, performed statistical analyses and prepared the manuscript. JS contributed to the design of this study and the interpretation of data. CX supported the scoring of immunohistochemical staining. All authors read and approved the submission of the manuscript.

\section{Acknowledgements}

The expert assistance of Drs. Tao Zhu and Mei Jin is greatly appreciated. This study was supported in part by Research Grant No. 2004BI 07 from the Health Department of Zhejiang Province, China (to L Wang) and NIH Grant CA92880 (to W Fan).

\section{References}

I. Gralow JR, Burstein HJ, Wood W, Hortobagyi GN, Gianni L, von Minckwitz G, Buzdar AU, Smith IE, Symmans WF, Singh B, Winer EP: Preoperative therapy in invasive breast cancer: pathologic assessment and systemic therapy issues in operable disease. J Clin Oncol 2008, 26:814-8I9.

2. Buzdar AU: Preoperative chemotherapy treatment of breast cancer-a review. Cancer 2007, I I 0:2394-2407.

3. Subramaniam DS, Isaacs C: Utilizing prognostic and predictive factors in breast cancer. Curr Treat Options Oncol 2005, 6: I 47-I 59.

4. Schmid P, Wischnewsky MB, Sezer O, Böhm R, Possinger K: Prediction of response to hormonal treatment in metastatic breast cancer. Oncology 2002, 63:309-316.

5. Horwitz KB, McGuire WL: Predicting response to endocrine therapy in human breast cancer: a hypothesis. Science 1975, 189:726-727.

6. Low SC, Dixon AR, Bell J, Ellis IO, Elston CW, Robertson JF, Blamey RW: Tumour oestrogen receptor content allows selection of elderly patients with breast cancer for conservative tamoxifen treatment. BrJ Surg 1992, 79:13|4-1316.

7. Van Poznak C, Tan L, Panageas KS, Arroyo CD, Hudis C, Norton L, Seidman AD: Assessment of molecular markers of clinical sensitivity to single-agent taxane therapy for metastatic breast cancer. J Clin Oncol 2002, 20:2319-2326.

8. Faneyte IF, Schrama JG, Peterse JL, Remijnse PL, Rodenhuis S, Vijver $M J$ van de: Breast cancer response to neoadjuvant chemotherapy: predictive markers and relation with outcome. $\mathrm{Br} J \mathrm{Can}$ cer 2003, 88:406-4I2.

9. Chhieng DC, Rodriguez-Burford C, Meleth S, Grizzle WE, Ferguson SM: Assessment of biomarker expression in predicting pathologic response to neoadjuvant chemotherapy in patients with locally advanced breast cancer. Breast J 2007, 13:534-535.

10. Bottini A, Berruti A, Bersiga A, Brizzi MP, Brunelli A, Gorzegno G, DiMarco B, Aguggini S, Bolsi G, Cirillo F, Filippini L, Betri E, Bertoli G, Alquati $P$, Dogliotti L: p53 but not bcl-2 immunostaining is predictive of poor clinical complete response to primary chemotherapy in breast cancer patients. Clin Cancer Res 2000, 6:275I-2758.

11. Makris A, Powles TJ, Dowsett M, Osborne CK, Trott PA, Fernando IN, Ashley SE, Ormerod MG, Titley JC, Gregory RK, Allred DC: Prediction of response to neoadjuvant chemoendocrine therapy in primary breast carcinomas. Clin Cancer Res 1997, 3:593-600.

12. Kuerer HM, Newman LA, Smith TL, Ames FC, Hunt KK, Dhingra K, Theriault RL, Singh G, Binkley SM, Sneige N, Buchholz TA, Ross MI, McNeese MD, Buzdar AU, Hortobagyi GN, Singletary SE: Clinical course of breast cancer patients with complete pathologic primary tumor and axillary lymph node response to doxorubicin-based neoadjuvant chemotherapy. J Clin Oncol 1999, 17:460-469.

13. Tomczykowski J, Szubstarski F, Kurylcio L, Stanislawek A, Barycki J, Baranowski W: Does the degree of cell lesion in breast cancer after inductive chemotherapy have any prognostic value? Acta Oncol 1999, 38:949-953.

14. Katzenellenbogen BS: Mechanisms of action and cross-talk between estrogen receptor and progesterone receptor pathways. J Soc Gynecol Investig 2000, 7(I SuppI):S33-37.

15. Mano MS, Rosa DD, De Azambuja E, Ismael GF, Durbecq V: The |7q|2-q2I amplification: Her2 and topoisomerase-Ilalpha and their importance to the biology of solid tumours. Cancer Treat Rev 2007, 33:64-77.

16. Volm M: Multidrug resistance and its reversal. Anticancer Res 1998, 18(4C):2905-2917.

17. Sataloff DM, Mason BA, Prestipino AJ, Seinige UL, Lieber CP, Baloch $\mathrm{Z}$ : Pathologic response to induction chemotherapy in locally advanced carcinoma of the breast: a determinant of outcome. J Am Coll Surg 1995, 180:297-306.

18. Sui M, Chen F, Chen Z, Fan W: Glucocorticoids interfere with therapeutic efficacy of paclitaxel against human breast and ovarian xenograft tumors. Int / Cancer 2006, | | 9:7| 2-7|7.

19. Kang SP, Martel M, Harris LN: Triple negative breast cancer: current understanding of biology and treatment options. Curr Opin Obstet Gynecol 2008, 20:40-46.

20. Chintamani , Singh JP, Mittal MK, Saxena S, Bansal A, Bhatia A, Kulshreshtha $P$ : Role of p-glycoprotein expression in predicting response to neoadjuvant chemotherapy in breast cancer-a prospective clinical study. World J Surg Oncol 2005, 3:61.

21. Kiang DT, Frenning DH, Goldman Al, Ascensao VF, Kennedy B]: Estrogen receptors and responses to chemotherapy and hormonal therapy in advanced breast cancer. $N$ Engl J Med 1978, 299:1330-1334.

22. Colleoni M, Minchella I, Mazzarol G, Nolè F, Peruzzotti G, Rocca A, Viale G, Orlando L, Ferretti G, Curigliano G, Veronesi P, Intra M, Goldhirsch A: Response to primary chemotherapy in breast cancer patients with tumors not expressing estrogen and progesterone receptors. Ann Oncol 2000, I I:1057-1059.

23. Berry DA, Cirrincione C, Henderson IC, Citron ML, Budman DR, Goldstein LJ, Martino S, Perez EA, Muss HB, Norton L, Hudis C, Winer EP: Estrogen-receptor status and outcomes of modern chemotherapy for patients with node-positive breast cancer. JAMA 2006, 295(14):1658-1667.

24. MacGrogan G, Mauriac L, Durand M, Bonichon F, Trojani M, de Mascarel I, Coindre JM: Primary chemotherapy in breast invasive carcinoma: predictive value of the immunohistochemical detection of hormonal receptors, p53, c-erbB-2, MiB I, pS2 and GST pi. Br J Cancer 1996, 74: I458- I 465

25. Stearns V, Singh B, Tsangaris T, Crawford JG, Novielli A, Ellis MJ, Isaacs C, Pennanen M, Tibery C, Farhad A, Slack R, Hayes DF: A prospective randomized pilot study to evaluate predictors of response in serial core biopsies to single agent neoadjuvant doxorubicin or paclitaxel for patients with locally advanced breast cancer. Clin Cancer Res 2003, 9(I): I24-|33.

26. Lippman ME, Allegra JC, Thompson EB, Simon R, Barlock A, Green L, Huff KK, Do HM, Aitken SC, Warren R: The relation between estrogen receptors and response rate to cytotoxic chemotherapy in metastatic breast cancer. N Engl J Med 1978, 298: $1223-1228$

27. Sui M, Huang Y, Park BH, Davidson NE, Fan W: Estrogen receptor alpha mediates breast cancer cell resistance to paclitaxel through inhibition of apoptotic cell death. Cancer Res 2007, 67:5337-5344.

28. Gapinski PV, Donegan WL: Estrogen receptors and breast cancer: prognostic and therapeutic implications. Surgery 1980 , 88:386-393.

29. Hilf R, Feldstein ML, Gibson SL, Savlov ED: The relative importance of estrogen receptor analysis as a prognostic factor for recurrence or response to chemotherapy in women with breast cancer. Cancer 1980, 45: 1993-2000.

30. Hilf R, Feldstein ML, Savlov ED, Gibson SL, Seneca B: The lack of relationship between estrogen receptor status and response to chemotherapy. Cancer 1980, 46(I 2 Suppl):2797-2800.

31. Minisini AM, Di Loreto C, Mansutti M, Artico D, Pizzolitto S, Piga A, Puglisi F: Topoisomerase Ilalpha and APE/ref-I are associated with pathologic response to primary anthracycline-based 
chemotherapy for breast cancer. Cancer Lett 2005, 224(I): 133-139.

32. Graham JD, Clarke CL: Physiological action of progesterone in target tissues. Endocr Rev 1997, 18:502-5I9.

33. Bouchard P: Progesterone and the progesterone receptor. J Reprod Med 1999, 44(2 Suppl):I53-I57.

34. Ross JS, Fletcher JA, Bloom KJ, Linette GP, Stec J, Symmans WF, Pusztai L, Hortobagyi GN: Targeted therapy in breast cancer: the HER2/neu gene and protein. Mol Cell Proteomics 2004, 3:379-398.

35. Traina A, Agostara B, Marasà L, Calabrò M, Zarcone M, Carruba G: HER2/neu expression in relation to clinicopathologic features of breast cancer patients. Ann N Y Acad Sci 2006 1089: I59-167.

36. Tiezzi DG, Andrade JM, Ribeiro-Silva A, Zola FE, Marana HR, Tiezzi MG: HER2, p53, p2I and hormonal receptors proteins expression as predictive factors of response and prognosis in locally advanced breast cancer treated with neoadjuvant docetaxel plus epirubicin combination. BMC Cancer 2007, 7:36.

37. Rody A, Karn T, Gätje R, Ahr A, Solbach C, Kourtis K, Munnes M, Loibl S, Kissler S, Ruckhäberle E, Holtrich U, von Minckwitz G, Kaufmann M: Gene expression profiling of breast cancer patients treated with docetaxel, doxorubicin, and cyclophosphamide within the GEPARTRIO trial: HER2, but not topoisomerase II alpha and microtubule-associated protein tau, is highly predictive of tumor response. Breast 2007, 16:86-93.

38. Warm M, Kates R, Mallmann P, Dick M, Nawroth F, Harbeck N, Paepke S, Thomas A: Impact of tumor biological factors on response to pre-operative epirubicin and paclitaxel chemotherapy in primary breast cancer. Anticancer Res 2007, 27:1031-1038.

39. Pegram MD, Finn RS, Arzoo K, Beryt M, Pietras RJ, Slamon DJ: The effect of HER2/neu overexpression on chemotherapeutic drug sensitivity in human breast and ovarian cancer cells. Oncogene 1997, 15:537-547.

40. Liedtke C, Mazouni C, Hess KR, André F, Tordai A, Mejia JA, Symmans WF, Gonzalez-Angulo AM, Hennessy B, Green M, Cristofanilli $M$, Hortobagyi GN, Pusztai L: Response to neoadjuvant therapy and long-term survival in patients with triple-negative breast cancer. J Clin Oncol 2008, 26: I275-I28I.

41. Keam B, Im SA, Kim HJ, Oh DY, Kim JH, Lee SH, Chie EK, Han W, Kim DW, Moon WK, Kim TY, Park IA, Noh DY, Heo DS, Ha SW, Bang Y]: Prognostic impact of clinicopathologic parameters in stage II/III breast cancer treated with neoadjuvant docetaxel and doxorubicin chemotherapy: paradoxical features of the triple negative breast cancer. BMC Cancer 2007, 7:203.

42. Carey LA, Dees EC, Sawyer L, Gatti L, Moore DT, Collichio F, Ollila DW, Sartor Cl, Graham ML, Perou CM: The triple negative paradox: primary tumor chemosensitivity of breast cancer subtypes. Clin Cancer Res 2007, 13:2329-2334.

43. Gurbuxani S, Zhou D, Simonin G, Raina V, Arya LS, Sazawal S, Marie $\mathrm{JP}$, Bhargava M: Expression of genes implicated in multidrug resistance in acute lymphoblastic leukemia in India. Ann Hematol 1998, 76:195-200.

44. Galimberti S, Testi R, Guerrini F, Fazzi R, Petrini M: The clinical relevance of the expression of several multidrug-resistantrelated genes in patients with primary acute myeloid leukemia. J Chemother 2003, 15:374-379.

45. Chintamani, Singh JP, Mittal MK, Saxena S, Bansal A, Bhatia A, Kulshreshtha P: Role of p-glycoprotein expression in predicting response to neoadjuvant chemotherapy in breast cancer-a prospective clinical study. World J Surg Oncol 2005, 3:6I.

46. Koh EH, Chung HC, Lee KB, Lim HY, Kim JH, Roh JK, Min JS, Lee KS, Kim BS: The value of immunohistochemical detection of $P$ glycoprotein in breast cancer before and after induction chemotherapy. Yonsei Med J 1992, 33:137-142.

47. Seymour L, Bezwoda WR, Dansey RD: P-glycoprotein immunostaining correlates with ER and with high Ki67 expression but fails to predict anthracycline resistance in patients with advanced breast cancer. Breast Cancer Res Treat 1995, 36:61-69.

48. MacGrogan G, Rudolph P, Mascarel Id I, Mauriac L, Durand M, Avril A, Dilhuydy JM, Robert J, Mathoulin-Pélissier S, Picot V, Floquet A, Sierankowski G, Coindre JM: DNA topoisomerase Ilalpha expression and the response to primary chemotherapy in breast cancer. BrJ Cancer 2003, 89:666-67I.

49. Durbecq V, Paesmans M, Cardoso F, Desmedt C, Di Leo A, Chan S, Friedrichs K, Pinter T, Van Belle S, Murray E, Bodrogi I, Walpole E,
Lesperance B, Korec S, Crown J, Simmonds P, Perren TJ, Leroy JY, Rouas G, Sotiriou C, Piccart M, Larsimont D: Topoisomerase-II alpha expression as a predictive marker in a population of advanced breast cancer patients randomly treated either with single-agent doxorubicin or single-agent docetaxel. Mol Cancer Ther 2004, 3:|207-12 |4.

50. Cardoso F, Durbecq V, Larsimont D, Paesmans M, Leroy JY, Rouas G, Sotiriou C, Renard N, Richard V, Piccart MJ, Di Leo A: Correlation between complete response to anthracycline-based chemotherapy and topoisomerase II-alpha gene amplification and protein overexpression in locally advanced/metastatic breast cancer. Int ] Oncol 2004, 24:20I-209.

5I. Villman K, Sjöström J, Heikkilä R, Hultborn R, Malmström P, Bengtsson NO, Söderberg M, Saksela E, Blomqvist C: TOP2A and HER2 gene amplification as predictors of response to anthracycline treatment in breast cancer. Acta Oncol 2006, 45:590-596.

\section{Pre-publication history}

The pre-publication history for this paper can be accessed here:

http://www.biomedcentral.com/1471-2407/9/226/pre pub
Publish with Bio Med Central and every scientist can read your work free of charge

"BioMed Central will be the most significant development for disseminating the results of biomedical research in our lifetime. "

Sir Paul Nurse, Cancer Research UK

Your research papers will be:

- available free of charge to the entire biomedical community

- peer reviewed and published immediately upon acceptance

- cited in PubMed and archived on PubMed Central

- yours - you keep the copyright
BioMedcentral 